\title{
Spinal intraarterial chemotherapy: interim results of a Phase I clinical trial
}

\author{
Athos Patsalides, MD, MPH, ${ }^{1}$ Yoshiya Yamada, MD, ${ }^{2}$ Mark Bilsky, MD, ${ }^{3}$ Eric Lis, MD, ${ }^{4}$ \\ Ilya Laufer, MD, ${ }^{3}$ and Yves Pierre Gobin, MD $^{1}$ \\ ${ }^{1}$ Interventional Neuroradiology, Department of Neurological Surgery, Weill Cornell Medical College; and Departments of \\ ${ }^{2}$ Radiation Oncology, ${ }^{3}$ Neurological Surgery, and ${ }^{4}$ Radiology, Memorial Sloan Kettering Cancer Center, New York, New York
}

OBJECTIVE Despite advances in therapies using radiation oncology and spinal oncological surgery, there is a subgroup of patients with spinal metastases who suffer from progressive or recurrent epidural disease and remain at risk for neurological compromise. In this paper the authors describe their initial experience with a novel therapeutic approach that consists of intraarterial $(\mathrm{IA})$ infusion of chemotherapy to treat progressive spinal metastatic disease.

METHODS The main inclusion criterion was the presence of progressive, metastatic epidural disease to the spine causing spinal canal compromise in patients who were not candidates for the standard treatments of radiation therapy and/ or surgery. All tumor histological types were eligible for this trial. Using the transfemoral arterial approach and standard neurointerventional techniques, all patients were treated with IA infusion of melphalan in the arteries supplying the epidural tumor. The protocol allowed for up to 3 procedures repeated at 3- to 6-week intervals. Outcome measures included physiological measures: 1) periprocedural complications according to the National Cancer Institute's Common Terminology Criteria for Adverse Events; and 2) MRI to assess for tumor response.

RESULTS Nine patients with progressive spinal metastatic disease and cord compression were enrolled in a Phase I clinical trial of selective IA chemotherapy. All patients had metastatic disease from solid organs and were not candidates for further radiation therapy or surgery. A total of 19 spinal intraarterial chemotherapy (SIAC) procedures were performed, and the follow-up period ranged from 1 to 7 months (median 3 months). There was 1 serious adverse event (febrile neutropenia). Local tumor control was seen in 8 of 9 patients, whereas tumor progression at the treated level was seen in 1 patient.

CONCLUSIONS These preliminary results support the hypothesis that SIAC is feasible and safe.

Clinical trial registration no.: NCT01637766 (clinicaltrials.gov)

http://thejns.org/doi/abs/10.3171/2015.5.SPINE14830

KEY WORDS intraarterial chemotherapy; spine; spinal metastasis; melphalan; oncology; technique

$\mathrm{S}$ PINE metastases from solid organ tumors are very common in the population of patients with cancer, and these lesions represent a major source of morbidity. Therapy for metastatic spine tumors is palliative, with the goals of maintaining or improving neurological function, providing mechanical stability, and achieving local tumor control. The majority of spinal solid organ tumor metastases are treated with radiation therapy alone or a combination of surgery and radiation. Local tumor control is typically dependent on the response to radia- tion when offered as definitive therapy or as a postoperative adjuvant. The integration of stereotactic radiosurgery (SRS) into treatment paradigms for metastatic tumors has demonstrated durable local control rates greater than $90 \%$ that are histology independent, providing better control than that observed with conventional external-beam radiation therapy. ${ }^{12,22}$ The availability of this treatment remains limited, however, and the vast majority of patients are still treated with conventional radiation therapy, with lower rates of local tumor control. Despite excellent tu-

ABBREVIATIONS IA = intraarterial; IV = intravenous; PLT = platelet; SAE = severe adverse event; SIAC = spinal intraarterial chemotherapy; SRS = stereotactic radiosurgery; $\mathrm{WBC}=$ white blood cell.

ACCOMPANYING EDITORIAL See pp 215-216. DOI: 10.3171/2015.6.SPINE15565.

SUBMITTED December 1, 2014. ACCEPTED May 13, 2015.

INCLUDE WHEN CITING Published online October 23, 2015; DOI: 10.3171/2015.5.SPINE14830. 
mor responses to radiation therapy, a subgroup of patients will demonstrate tumor progression, leaving them with no effective treatment options and placing them at risk for progressive spinal cord or cauda equina compression and paralysis. Recent experience with intraarterial (IA) chemotherapy for retinoblastoma ${ }^{2}$ led us to investigate the possibility of delivering IA chemotherapy to spine tumors in instances in which surgical and radiation options were exhausted. This paper presents interim data from a Phase I clinical trial aimed to test the hypothesis that spinal intraarterial chemotherapy (SIAC) can be safely applied in patients with metastatic spinal epidural tumors.

\section{Methods}

\section{Patient Characteristics}

This single-arm, progressive, Phase I clinical trial was approved by the Weill Cornell Medical College Institutional Review Board and is listed on the clinical trials website (Intra-arterial Chemotherapy for Spinal Metastases, www. clinicaltrials.gov; NCT01637766). A physician-sponsored Investigational New Drug approval (No. 113342) was obtained from the FDA. The inclusion and exclusion criteria are described in Table 1. All tumor histological types were eligible for this treatment. Nine patients (5 male; age range 19-76 years, median age 48 years) were enrolled in the trial between July 2009 and June 2012. Informed consent was obtained from all patients.

All patients had MRI evidence of progressive spinal epidural disease, and had already been treated with surgery and SRS at that level. Each patient's history, clinical condition, and imaging were reviewed at the spinal tumor board, and the consensus among neurosurgery and radiation oncology experts was that these patients were not candidates for further surgery or radiation. All patients underwent spinal MRI scans with contrast within 4 weeks of study enrollment. The grading of epidural disease and spinal canal compromise was based on axial MR images, using

\section{TABLE 1. Inclusion and exclusion criteria for patients with} progressive spinal epidural tumor

\begin{tabular}{l} 
Inclusion criteria \\
Patients w/ progressive metastatic disease to the spine causing \\
cord compression Grades $1 \& 2$ who are not candidates for the \\
standard treatment of radiation therapy or surgery \\
Patients older than 18 yrs \\
Patients able to give informed consent \\
Exclusion criteria \\
MRI findings of Grade 3 epidural cord compression \\
Vascular supply to the spinal cord from the same segmental arter- \\
ies supplying the tumor (angiographic exclusion criterion) \\
\hline Life expectancy $<3$ mos \\
Pregnant or lactating patients \\
Female patients w/ inadequate contraception \\
\hline History of severe allergy to contrast media \\
\hline Renal insufficiency (creatinine $>1.5$ mg/dl) \\
\hline WBCs $<3000$ cells $/ \mathrm{mm}^{3}$ \\
\hline PLTs $<75,000$ cells $/ \mathrm{mm}^{3}$ \\
\hline
\end{tabular}

the following classification for epidural spinal cord compression: Grade 0, no subarachnoid space compression; Grade 1, subarachnoid space partially obliterated without cord compression; Grade 2, subarachnoid space partially obliterated with cord compression; and Grade 3, subarachnoid space completely blocked, with severe cord compression. ${ }^{4}$ Only patients with Grades 1 and 2 were eligible for the trial. Exclusion criteria were Grade 3 spinal cord/ cauda equina compression, rapidly worsening neurological symptoms, low white blood cell (WBC) counts, and life expectancy less than 3 months. In patients with multifocal spinal epidural disease, the target area was the one with the most significant degree of epidural disease based on MRI. The primary objective of the trial was to evaluate the safety and feasibility of SIAC, and the secondary objective was to evaluate changes in epidural tumor size after treatment. The FDA approved enrollment of up to 20 patients. The study was to be discontinued if a severe adverse event (SAE) occurred in more than 3 of the first 10 patients.

\section{The SIAC Procedure}

The spinal arterial catheterization was performed using standard interventional techniques via femoral arterial access. Either conscious sedation or general anesthesia was used, primarily depending on the patient's ability to lie flat for the duration of the procedure. The treatment area was selected based on the MRI scans. Epidural tumor was seen as enhancing area on T1-weighted sequences and high-signal-intensity area on T2-weighted sequences. The arterial territory to be treated with chemotherapy was determined by following basic knowledge of the spinal arterial anatomy, so that the entire targeted area was covered. The segmental (intercostal or lumbar) arteries supplying the epidural tumor at the level of compression, including at least one vertebral body level above and one vertebral body level below, were catheterized and studied with digital subtraction angiography. Patients with vascular supply to the spinal cord (anterior and/or posterior spinal arteries) originating from the same segmental arteries supplying the tumor were excluded from the study and chemotherapy was not administered (angiographic exclusion criterion). Following diagnostic angiography, a microcatheter was used to superselectively catheterize the ventral branch of the segmental arteries and permanently occlude it with detachable coils. This step was undertaken to minimize the flow of chemotherapy into the normal paraspinal soft tissues and the thoracic or abdominal wall, thus allowing for maximum delivery of chemotherapy to the vessels of the dorsal branch supplying the tumor. Then, chemotherapy was infused through the microcatheter using a pulsatile injection technique to avoid streamlining and heterogeneous delivery. ${ }^{9}$

\section{Chemotherapy Agent}

The chemotherapy agent used in this study was melphalan (brand name Alkeran). The total dose injected was the recommended systemic dose of $16 \mathrm{mg} / \mathrm{m}^{2}$, adjusted for WBCs, platelets (PLTs), and renal function per FDA guidelines. Melphalan was diluted with normal saline to achieve a 1:1 concentration, and the infusate was equally 
divided among the segmental arteries supplying the targeted tumor.

\section{Postprocedure Evaluation and Follow-Up}

The study patients were observed for 5 hours after the procedure prior to discharge to home, and then followed weekly with physical examinations and complete blood counts. All adverse events within 30 days of the last SIAC were recorded. The possible adverse events were classified as catheterization- or angiography-related (i.e., vessel dissection, vessel thrombosis or occlusion, renal insufficiency, contrast medium allergy, hematoma, death); local chemotherapy toxicity (i.e., spinal cord ischemia or toxicity, tissue or skin necrosis); and systemic chemotherapy toxicity (i.e., myelosuppression, nausea, vomiting, sepsis, death). Serious adverse events were defined as Grade 3 or greater toxicity according to the National Cancer Institute's Common Terminology Criteria for Adverse Events, version 4.03 (2010).

Enrollment to the trial required at least 1 SIAC, but the protocol allowed for up to 3 SIAC procedures at the same level, repeated at 3-6 week intervals based on the results of complete blood counts. The patients were followed with blood tests and office visits for at least 30 days after the last treatment. As part of the protocol, a spinal MRI study with contrast was performed approximately 4 weeks after the last SIAC treatment to assess for complications related to the experimental treatment (hemorrhage, spinal cord inflammation, spinal cord infarct) as well as tumor size changes. We made every possible effort to obtain MRI scans at later time intervals, and any spinal imaging thereafter was performed as clinically indicated (not required by the protocol). The follow-up duration was determined according to the last available spinal MRI scan. All MRI scans were reviewed by an experienced neuroradiologist (E.L.), and any measurable increase in the dimension of epidural disease or increased mass effect on the thecal sac, spinal cord, or cauda equina was considered evidence of disease progression.

\section{Results}

Metastatic tumor histological diagnoses included colorectal carcinoma in 3 patients, and melanoma, renal cell carcinoma, lung carcinoma, leiomyosarcoma, Ewing's sar- coma, and hemangiopericytoma in 1 patient each (Table 2). The targeted tumor was located in the thoracic spine in 7 patients and in the lumbar spine in 2 patients. A total of 19 procedures were performed in 9 patients (median 2 procedures per patient). Two experienced interventional neuroradiologists (A.P., Y.P.G.) performed the SIAC procedures. The goal was for each patient to receive 3 separate treatments, but only 4 patients received all 3 IA infusions. Two patients received 2 IA infusions and 3 received a single IA infusion. Reasons for patients not receiving the goal number of infusions included the following: SAE after initial treatment $(\mathrm{n}=1)$, local tumor progression representing failure $(\mathrm{n}=1)$, patient availability $(\mathrm{n}=1)$, and significant clinical deterioration due to systemic disease progression $(\mathrm{n}=2)$. A follow-up spinal MRI study with contrast at 4 weeks postprocedure as per the protocol was obtained in all patients. Three patients had more than one follow-up MRI session. The follow-up duration ranged from 1 to 7 months (median 3 months, mean 3.3 months).

No catheterization-related, angiography-related, or local toxicity adverse events were observed. One patient developed neutropenic fevers and was admitted to the hospital for prophylactic antibiotics and supportive care (Grade 4 adverse event). This patient had diffuse Ewing's sarcoma and had previously received multiple regimens of aggressive chemotherapy and extensive spinal radiation. This was the only SAE in the trial. Another patient developed nausea after the procedure (Grade 2 adverse event); this did not qualify as an SAE.

Local tumor control was achieved in 8 of 9 patients. Based on the MRI scans obtained after the last SIAC treatment, the tumor remained stable in 7 patients and slightly decreased in size in 1 patient. Among the 8 patients with local tumor control, 7 had imaging evidence of disease progression at other spinal sites outside the SIAC-treated levels (Fig. 1). Tumor progression at the treated level was seen only in the patient with leiomyosarcoma; that tumor had a large paraspinal as well as epidural component that continued to increase in size despite 2 technically successful IA chemotherapy sessions. Therefore, the decision was made not to proceed with further SIAC.

\section{Discussion}

In this paper we describe our initial experience with 19 procedures of SIAC in 9 patients. The primary feasibility

TABLE 2. Characteristics of 9 patients with progressive spinal metastatic tumors

\begin{tabular}{clccclll}
\hline $\begin{array}{c}\text { Age (yrs), } \\
\text { Sex }\end{array}$ & Primary Tumor & $\begin{array}{c}\text { Treated } \\
\text { Level }\end{array}$ & $\begin{array}{c}\text { No. of IA } \\
\text { Treatments }\end{array}$ & $\begin{array}{c}\text { Follow-Up } \\
\text { (mos) }\end{array}$ & $\begin{array}{c}\text { Results at } \\
\text { Treated Level }\end{array}$ & $\begin{array}{c}\text { Tumor Progression } \\
\text { at Other Sites }\end{array}$ & Complications \\
\hline 58, F & Colorectal carcinoma & L-4 & 3 & 5 & Stable & Spine & None \\
\hline 56, F & Melanoma & T-2 & 1 & 1 & Stable & Spine, systemic & None \\
\hline 45, M & Colorectal carcinoma & T-7 & 3 & 2 & Stable & Spine, systemic & None \\
\hline 19, M & Ewing's sarcoma & T10-11 & 1 & 3 & Improved & Spine, systemic & Febrile neutropenia \\
\hline $76, \mathrm{~F}$ & Leiomyosarcoma & T-10 & 2 & 3 & Progressed & Spine, systemic & None \\
\hline $65, \mathrm{M}$ & Colorectal carcinoma & T12-L1 & 3 & 4 & Stable & Systemic & None \\
\hline $48, \mathrm{M}$ & Renal cell carcinoma & L2-3 & 1 & 2 & Stable & Spine, systemic & None \\
\hline $36, \mathrm{~F}$ & Hemangiopericytoma & T1-2 & 2 & 3 & Stable & Spine, systemic & None \\
\hline $39, \mathrm{M}$ & Lung carcinoma & T7-8 & 3 & 7 & Stable & Spine, systemic & None \\
\hline
\end{tabular}



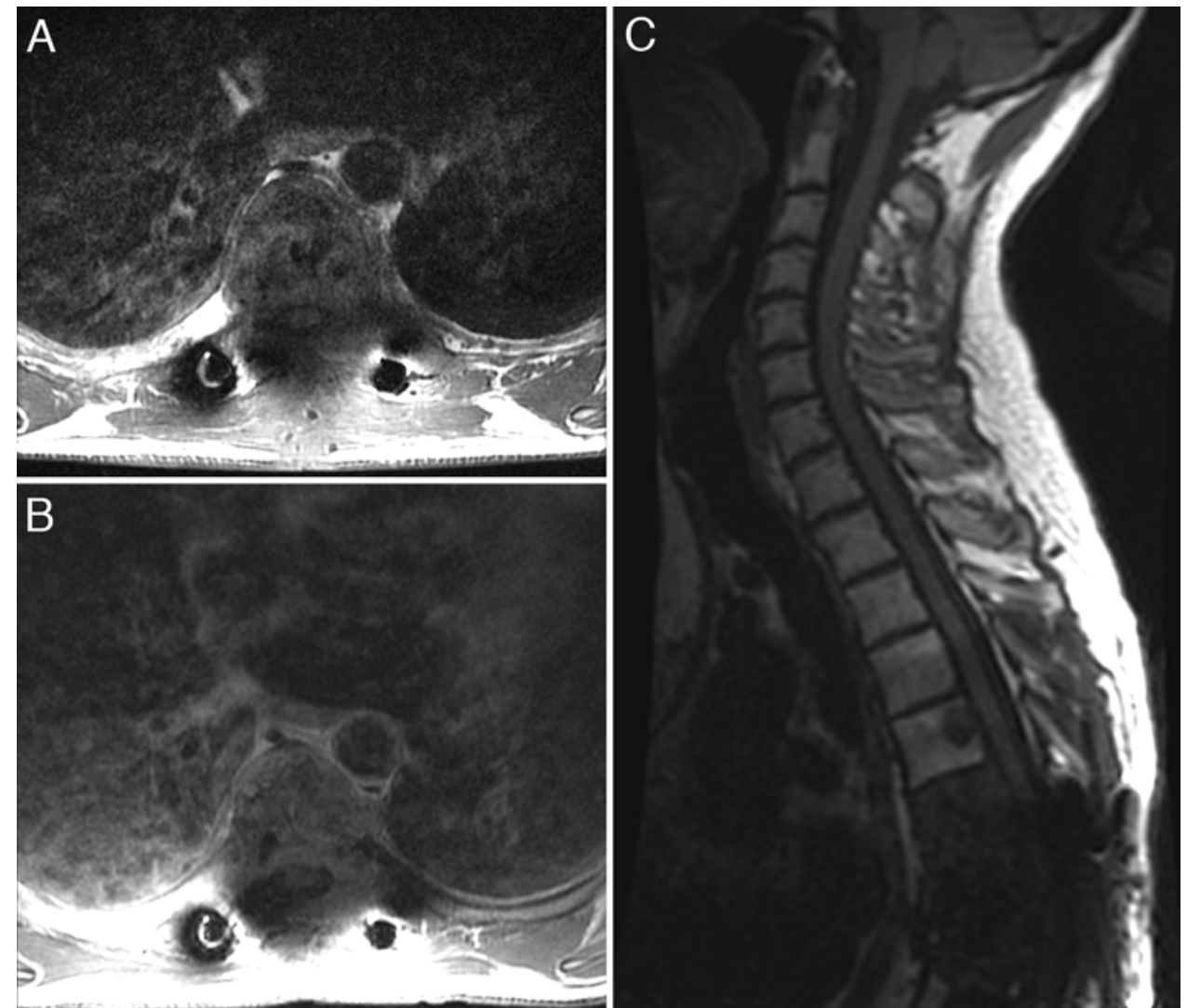

FIG. 1. Neuroimages obtained in a 39-year-old man with metastatic lung carcinoma to the T-7 level. A: Axial postcontrast T1-weighted MR image shows recurrent epidural disease after repeated surgery and SRS. B: Follow-up axial postcontrast T1-weighted MR image obtained after 3 cycles of IA chemotherapy shows stable disease 7 months after treatment. C: Sagittal T1-weighted MR image shows new metastatic disease at the T-5 level (not treated with IA chemotherapy). This patient also had disease progression in the lungs (not shown).

end point was reached in all 9 patients and 19 procedures, and the primary safety end point was reached in 8 of 9 patients. Even though the trial is still open, we decided to report interim results because we wanted to increase awareness for this procedure that in our opinion has a role to play in the management of selected patients with spinal metastatic disease. Because of the exceptional results of radiation therapy at our institution, recruitment has been slow and it may take years to complete the trial. To our knowledge, this is the first report of a prospective clinical trial of IA chemotherapy for spinal epidural disease. Chiras et al. described a series of 25 patients with metastatic disease to the spinal column $(n=10)$, iliac bone and sacrum $(\mathrm{n}=15)$ who were treated with transarterial chemoembolization using polyvinyl alcohol particles, pirarubicin, and carboplatin, achieving significant pain relief in 15 patients and local tumor control in 16 patients with available imaging follow-up. ${ }^{6}$ There are two main differences between this protocol and ours: 1) our technique does not involve the use of embolic agents and consists of chemotherapy infusion only; and 2) the focus of our treatment is the epidural disease and neurological symptoms as opposed to pain levels and overall tumor size.

Advances in the field of interventional radiology during the last 2 decades have led to the use of selective IA chemotherapy for the treatment of malignant diseases. Conventionally, the systemic dose of an anticancer drug is increased to the maximum tolerated dose, assuming that higher drug concentrations will be achieved, which in turn will result in increased effectiveness. Intraarterial (or other regional) drug delivery may be used to circumvent the maximum tolerated dose limitation and achieve an even higher drug concentration at the target area. A prominent example of the effectiveness of regional drug delivery is found in the treatment of liver malignancies, where IA chemotherapy (with or without embolization) has been used as a palliative treatment for unresectable hepatocellular carcinoma, as neoadjuvant therapy prior to orthotropic liver transplantation, and as first-line treatment for unresectable hepatic metastases from colorectal cancer.,14,15

The pharmacokinetic advantage of administering chemotherapy through the IA route is that the first pass of the drug is through the tumor vascular bed, thus avoiding the first-pass metabolism of chemotherapeutic agents administered orally or intravenously. Therefore, the local concentration of chemotherapy in the tumor is very high, with less systemic exposure and possibly less systemic toxicity. After the first pass of the drug through the tumor vascular bed, the remaining drug leaves the target area and enters the systemic circulation. Even though there is a component of the drug that behaves as if it were injected intravenously, the resulting intravenous (IV) dose is much less than the dose delivered with conventional IV systemic dosing. The maximum effect in terms of increased drug 
concentration at the target area is achieved with drugs that have high total body clearance and low regional exchange rates at the target area. ${ }^{7}$ Other desirable characteristics for a drug delivered intraarterially are a linear dose-response effect (i.e., tumor cytotoxicity proportional to the level of exposure), minimal nontarget regional toxicity, and minimal systemic toxicity.

The advantage of IA drug administration has been shown in the liver, where therapeutic drug concentrations within liver tumor were up to 100 times greater than those achievable with systemic chemotherapy, ${ }^{11,16,19}$ and in the CNS, where IA administration of technetium-99m hexylmethylpropylene amineoxine into human cerebral arteries achieved a 50 times higher concentration in brain tissue compared with IV injection of the same substance. ${ }^{17} \mathrm{Com}-$ parative pharmacokinetics of gemcitabine during IA and IV delivery in patients with unresectable pancreatic cancer showed significantly lower plasma concentrations of gemcitabine and its deaminated metabolite in the systemic circulation after IA delivery, suggestive of a major advantage of IA delivery in terms of reduced systemic toxicity. ${ }^{20}$

The ideal tumor to be treated with IA chemotherapy is a regionally confined lesion, with an arterial supply that is feasible and safe to catheterize, and in which local tumor control or regression is clinically meaningful. Spinal epidural metastatic disease fits all these criteria. All patients enrolled in the trial had imaging evidence of progressive recurrent epidural disease and were not eligible for the standard treatments. These patients, who are destined to develop cord/cauda equina compression and neurological compromise, are the focus of this novel therapeutic approach, with SIAC aiming to achieve local tumor control and prevent the devastating complication of spinal cord or cauda equina compression.

The goal of IA drug delivery is to increase the effectiveness of a drug that has already demonstrated some activity against the targeted disease. Melphalan is an alkylating agent currently used systemically to treat multiple myeloma and ovarian cancer. Melphalan has also been used intraarterially with good results for the treatment of limb melanoma (using isolated limb perfusion), ${ }^{5,13,21}$ for the treatment of colorectal metastatic disease to the liver (chemoembolization), and it has also shown effectiveness against lung metastases ${ }^{8,10}$ and soft-tissue sarcoma. ${ }^{5} \mathrm{Be}-$ cause melanoma and colorectal carcinoma are relatively radioresistant tumors, we expected that these histological types would be common in our cohort.

Melphalan is an excellent choice for IA infusion because it has rapid elimination and high total body clearance (half-life of 60-90 minutes), largely independent of renal and hepatic function. In addition, melphalan is a cell-cycle-nonspecific alkylating agent with a linear doseresponse curve, which is advantageous for regional drug delivery. A systemic dose of $16 \mathrm{mg} / \mathrm{m}^{2}$ is considered "lowdose" for multiple myeloma protocols and is usually well tolerated, with acceptable and predictable systemic toxicity. The most common side effect of melphalan is bone marrow suppression; the WBC and PLT count nadirs usually occur 2-3 weeks after treatment, and recover 2-3 weeks later. Melphalan has no neurotoxicity. Intraarterial administration of melphalan in the ophthalmic artery has revolutionized the treatment of intraocular retinoblastoma. ${ }^{1}$ To date, we have treated more than 150 patients using this novel approach. Based on prior experience with selective IA chemotherapy for the treatment of ocular retinoblastoma, ${ }^{2}$ and the high local control rates achieved with selective IA injection of chemotherapy in recurrent limb melanoma, ${ }^{18}$ we postulated that SIAC with selective injection of melphalan in the arteries feeding the metastatic disease would be feasible and safe and might prove beneficial in achieving local control of the spinal tumor.

The safety end point in our study was met in 8 of 9 patients. Great care was taken to avoid infusion of the chemotherapy agent into a segmental artery that supplies the spinal cord, which could potentially result in spinal cord toxicity and myelitis. No toxicity to the spinal cord was exhibited in this study. One patient developed severe bone marrow suppression and febrile neutropenia from systemic drug toxicity. This patient had been heavily treated with IV chemotherapy and spinal radiation in the past and was at a higher than usual risk for bone marrow suppression. In general, however, systemic toxicity is predictable when using body surface or weight dosing schemes, and adjusting the total dose according to WBC and PLT counts and renal function can prevent severe bone marrow suppression.

The secondary objective of this trial is to assess clinical response and epidural tumor changes after SIAC. The majority of patients in the trial had no evidence of disease progression at the treated level after SIAC, despite definitive evidence of progressive/relapsing disease prior to enrollment, and prior surgical and radiation treatments. Because all patients had recurrent progressive epidural disease without options for further radiation or surgery, it's conceivable that without any intervention the tumors would probably continue to progress. Obviously, the tumor response needs to be studied and validated in a large multicenter trial with randomization. Although there is evidence of the clinical effectiveness of melphalan against a variety of malignancies, the choice of a single therapeutic agent for all tumor histological types is not optimal. Similarly to our approach, Chiras et al. used a single therapeutic agent for treating bone metastatic disease caused by a variety of tumors. ${ }^{6}$ Ideally, each tumor could have been targeted with a separate chemotherapy agent, but this approach requires separate protocols, investigational new drug approvals, and trials for each drug. Given the low rate of accrual and the strict indications, it would take many years before such trials would be completed. On the other hand, the preliminary good clinical results of this study across a variety of different histological types suggest that SIAC is effective against epidural metastatic disease. The fact that in most patients there were imaging findings of tumor progression at other-untreated with SIAC-spinal levels supports the notion that local tumor control at the treated level was due to SIAC.

The very small study population and the short duration of follow-up are limitations of our study. The patients were enrolled in our trial after they had exhausted the standard treatment options, and most had reached terminal stages of their disease. The follow-up duration was based on the last available MRI scan, and we made every effort to follow the patient until they died of systemic disease or were 
admitted to hospice. This trial is aimed to look at safety and feasibility, and the short follow-up does not affect the main objective of a Phase I trial (i.e., safety and feasibility). A future trial with effectiveness as an end point will need to have longer follow-up times to have credible results for local tumor control. We believe, however, that even if the survival proves to be on the order of few months in a larger series of patients, preventing or delaying inevitable cord/cauda equina compression is still very important in improving the quality of life and decreasing the stress and burden of severe neurological deficits on the patients and their families.

\section{Conclusions}

The interim results of this Phase I clinical trial support the hypothesis that SIAC with melphalan for patients with recurrent epidural metastatic disease is feasible and safe. The encouraging results in local tumor control suggest that this may become an important tool for the palliative treatment of patients in whom radiation therapy and surgery have failed. The results thus far support continuing the Phase I trial.

\section{References}

1. Abramson DH, Dunkel IJ, Brodie SE, Kim JW, Gobin YP: A phase I/II study of direct intraarterial (ophthalmic artery) chemotherapy with melphalan for intraocular retinoblastoma initial results. Ophthalmology 115:1398-1404, 1404.e1, 2008

2. Abramson DH, Francis JH, Dunkel IJ, Marr BP, Brodie SE, Gobin YP: Ophthalmic artery chemosurgery for retinoblastoma prevents new intraocular tumors. Ophthalmology 120:560-565, 2013

3. Anand A, Anand N, Anand A: Re: Reappraisal of hepatic arterial infusion in the treatment of nonresectable liver metastases from colorectal cancer. J Natl Cancer Inst 88:838839, 1996

4. Bilsky MH, Laufer I, Fourney DR, Groff M, Schmidt MH, Varga PP, et al: Reliability analysis of the epidural spinal cord compression scale. J Neurosurg Spine 13:324-328, 2010

5. Brady MS, Brown K, Patel A, Fisher C, Marx W: A phase II trial of isolated limb infusion with melphalan and dactinomycin for regional melanoma and soft tissue sarcoma of the extremity. Ann Surg Oncol 13:1123-1129, 2006

6. Chiras J, Adem C, Vallée JN, Spelle L, Cormier E, Rose M: Selective intra-arterial chemoembolization of pelvic and spine bone metastases. Eur Radiol 14:1774-1780, 2004

7. Collins JM: Pharmacologic rationale for regional drug delivery. J Clin Oncol 2:498-504, 1984

8. Den Hengst WA, Hendriks JM, Van Hoof T, Heytens K, Guetens G, de Boeck G, et al: Selective pulmonary artery perfusion with melphalan is equal to isolated lung perfusion but superior to intravenous melphalan for the treatment of sarcoma lung metastases in a rodent model. Eur J Cardiothorac Surg 42:341-347, 2012

9. Gobin YP, Cloughesy TF, Chow KL, Duckwiler GR, Sayre JW, Milanese K, et al: Intraarterial chemotherapy for brain tumors by using a spatial dose fractionation algorithm and pulsatile delivery. Radiology 218:724-732, 2001

10. Hendriks JM, Grootenboers MJ, Schramel FM, van Boven WJ, Stockman B, Seldenrijk CA, et al: Isolated lung perfusion with melphalan for resectable lung metastases: a phase I clinical trial. Ann Thorac Surg 78:1919-1927, 2004

11. Konno T: Targeting cancer chemotherapeutic agents by use of lipiodol contrast medium. Cancer 66:1897-1903, 1990
12. Laufer I, Iorgulescu JB, Chapman T, Lis E, Shi W, Zhang Z, et al: Local disease control for spinal metastases following "separation surgery" and adjuvant hypofractionated or highdose single-fraction stereotactic radiosurgery: outcome analysis in 186 patients. J Neurosurg Spine 18:207-214, 2013

13. Lens MB, Dawes M: Isolated limb perfusion with melphalan in the treatment of malignant melanoma of the extremities: a systematic review of randomised controlled trials. Lancet Oncol 4:359-364, 2003

14. Lesurtel M, Müllhaupt B, Pestalozzi BC, Pfammatter T, Clavien PA: Transarterial chemoembolization as a bridge to liver transplantation for hepatocellular carcinoma: an evidencebased analysis. Am J Transplant 6:2644-2650, 2006

15. Molinari M, Kachura JR, Dixon E, Rajan DK, Hayeems EB, Asch MR, et al: Transarterial chemoembolisation for advanced hepatocellular carcinoma: results from a North American cancer centre. Clin Oncol (R Coll Radiol) 18:684-692, 2006

16. Nakamura H, Hashimoto T, Oi H, Sawada S: Transcatheter oily chemoembolization of hepatocellular carcinoma. Radiology 170:783-786, 1989

17. Namba H, Kobayashi S, Iwadate Y, Saegusa T, Sato A, Watanabe Y, et al: Assessment of the brain areas perfused by superselective intra-arterial chemotherapy using single photon emission computed tomography with technetium-99m-hexamethyl-propyleneamine oxime--technical note. Neurol Med Chir (Tokyo) 34:832-835, 1994

18. Noorda EM, Vrouenraets BC, Nieweg OE, van Geel BN, Eggermont AM, Kroon BB: Isolated limb perfusion for unresectable melanoma of the extremities. Arch Surg 139:12371242, 2004

19. Ramsey DE, Kernagis LY, Soulen MC, Geschwind JF: Chemoembolization of hepatocellular carcinoma. J Vasc Interv Radiol 13:S211-S221, 2002

20. Shamseddine AI, Khalifeh MJ, Mourad FH, Chehal AA, Al-Kutoubi A, Abbas J, et al: Comparative pharmacokinetics and metabolic pathway of gemcitabine during intravenous and intra-arterial delivery in unresectable pancreatic cancer patients. Clin Pharmacokinet 44:957-967, 2005

21. Thompson JF, Gianoutsos MP: Isolated limb perfusion for melanoma: effectiveness and toxicity of cisplatin compared with that of melphalan and other drugs. World J Surg 16:227-233, 1992

22. Yamada Y, Bilsky MH, Lovelock DM, Venkatraman ES, Toner S, Johnson J, et al: High-dose, single-fraction imageguided intensity-modulated radiotherapy for metastatic spinal lesions. Int J Radiat Oncol Biol Phys 71:484-490, 2008

\section{Disclosure}

Dr. Yamada is a consultant for Varian Medical Systems. Dr. Laufer is a consultant for SpineWave and DePuy/Synthes.

\section{Author Contributions}

Conception and design: Patsalides, Yamada, Bilsky, Gobin. Acquisition of data: Patsalides. Analysis and interpretation of data: Patsalides, Lis. Drafting the article: Patsalides. Critically revising the article: Patsalides, Bilsky. Reviewed submitted version of manuscript: all authors. Approved the final version of the manuscript on behalf of all authors: Patsalides. Study supervision: Patsalides.

\section{Correspondence}

Athos Patsalides, Interventional Neuroradiology, Department of Neurological Surgery, Weill Cornell Medical College, 525 E. 68th St., Box 99, New York, NY 10065. email: atp9002@med. cornell.edu. 